\title{
Momentum transfer to interplanetary dust from the solar wind
}

\author{
T. Minato ${ }^{1,2,3}$, M. Köhler ${ }^{3}$, H. Kimura ${ }^{3}$, I. Mann ${ }^{3}$, and T. Yamamoto ${ }^{1,2}$ \\ ${ }^{1}$ Department of Earth and Planetary Sciences, Nagoya University, Nagoya 464-8604, Japan \\ e-mail: minato@eps.nagoya-u.ac.jp \\ 2 Institute of Low Temperature Science, Hokkaido University, Sapporo 060-0819, Japan \\ 3 Institut für Planetologie, Westfälische Wilhelms-Universität, Wilhelm-Klemm-Straße 10, 48149 Münster, Germany
}

Received 7 May 2004 / Accepted 14 July 2004

\begin{abstract}
Solar-wind particles striking dust grains in orbit around the sun exert not only a repulsive force on the grains, but also a drag force, called the plasma or pseudo Poynting-Robertson (PR) effect, which limits their dynamical lifetimes. To better understand the dynamical evolution of interplanetary dust, we study the momentum transfer from the solar wind to interplanetary dust taking into account the passage of impinging particles through the grains. The pseudo PR drag is one of the most important perturbations for the dynamics of interplanetary dust of any sizes. However, previous studies underestimated the lifetimes of small grains by overestimating the acting solar-wind forces. Our study also provides the velocity distribution of neutralized solar-wind particles after passage through a grain along with some implications for the generation of pick-up ions by solar-wind interaction with dust grains.
\end{abstract}

Key words. interplanetary medium - meteors, meteoroids - solar wind

\section{Introduction}

The impinging of solar-wind particles on a dust grain moving around the sun exerts a force on the grain in the directions parallel and perpendicular to the solar-wind flow. The perpendicular component decreases the orbital angular momentum and energy of the grain. This limits the lifetime of the grain in a manner similar to the Poynting-Robertson (PR) effect, which results from its interaction with electromagnetic radiation (Whipple 1955). This tangential force due to the solar-wind impact is hereafter referred to as the pseudo PR effect analogous to the PR effect. The pseudo PR effect is comparable to the PR effect for grains larger than $0.1 \mu \mathrm{m}$ and exceeds the PR effect for grains smaller than $0.1 \mu \mathrm{m}$ (Burns et al. 1979; Mukai \& Yamamoto 1982). The magnitude of direct solar-wind pressure may exceed that of direct radiation pressure for $0.01 \mu \mathrm{m}$ grains (Mukai \& Yamamoto 1982). Consequently, the direct solar-wind pressure and the pseudo PR drag play a key role in the dynamical evolution of small dust grains around the sun.

Previous studies of solar-wind forces assumed that impinging solar-wind particles are perfectly reflected or absorbed on the grain surfaces (Mukai \& Yamamoto 1982; Gustafson 1994). However, the solar-wind particles can pass through small dust grains. As a result of this "small-particle effect", a fraction of their momentum is not transferred to grains smaller than the distance that the particles can travel in matter. As we show in this paper, the small-particle effect limits the direct solar-wind pressure and the pseudo PR effect for small grains.

Large dust particles interacting with solar-wind particles are discussed to generate neutral atoms with increasing rates toward the sun (Banks 1971; Fahr et al. 1981). The slow moving neutrals are ionized by solar UV and subsequently picked up by the solar-wind's frozen-in magnetic field, which redistributes them in the solar wind. It is suggested that the neutrals generated at distances $r \leq 1 \mathrm{AU}$ from the sun provide a source of pick-up ions measured at $r \geq 1$ AU (Gloeckler \& Geiss 2001). Our study provides estimates for the velocity distribution of solar-wind particles after passage through dust grains

Here we study the momentum transfer of solar-wind particles to dust grains taking into account the passage of the particles through the grains. Implications for solar-wind forces on dust grains and the role of dust in the formation of pick-up ions are discussed.

\section{Interaction of dust with solar-wind particles}

\subsection{Momentum transfer cross section}

The contribution of solar-wind forces to the motion of a dust grain with mass $m$ is described as (Burns et al. 1979)

$m \dot{v}=F_{\mathrm{sw}}\left[\left(1-\frac{2 \dot{r}}{v_{\mathrm{sw}}}\right) \boldsymbol{e}_{r}-\left(\frac{r \dot{\theta}}{v_{\mathrm{sw}}}\right) \boldsymbol{e}_{\theta}\right]$,

where $\boldsymbol{v}$ is the velocity of the grain $\boldsymbol{v}=\dot{\boldsymbol{r}} \boldsymbol{e}_{r}+r \dot{\boldsymbol{\theta}} \boldsymbol{e}_{\theta}$ and $v_{\mathrm{sw}}$ denotes the heliocentric solar-wind speed. The force $F_{\mathrm{sw}}$ is given by

$F_{\mathrm{sw}}=\sum_{i} \rho_{i} v_{\mathrm{sw}}^{2} C_{\mathrm{sw}, i}$,

where $\rho_{i}$ is the mass density of the $i$ th species of solar-wind particles, which consist of mainly protons and $\alpha$-particles. 
Table 1. The projected range $l_{i}$ and the stopping cross section $S_{i}^{(1)}$ of protons $(i=\mathrm{p})$ and $\alpha$-particles $(i=\alpha)$ with initial kinetic energy $1 \mathrm{keV} / \mathrm{amu}$ for $\mathrm{SiO}_{2}$ and carbon (Mikheev et al. 1993; Eder et al. 1997; Bauer et al. 1998; Møller et al. 2002).

\begin{tabular}{lcccc}
\hline \hline & $\begin{array}{c}l_{\mathrm{p}} \\
(\mu \mathrm{m})\end{array}$ & $\begin{array}{c}l_{\alpha} \\
(\mu \mathrm{m})\end{array}$ & $\begin{array}{c}S_{\mathrm{p}}^{(1)} \\
\left(10^{-15} \mathrm{eV} \mathrm{cm}^{2}\right)\end{array}$ & $\begin{array}{c}S_{\alpha}^{(1)} \\
\left(10^{-15} \mathrm{eV} \mathrm{cm}^{2}\right)\end{array}$ \\
\hline $\mathrm{SiO}_{2}$ & 0.092 & 0.16 & 8.2 & 19.0 \\
Carbon & 0.067 & 0.14 & 3.0 & 5.9 \\
\hline
\end{tabular}

Because $\rho_{i} v_{\mathrm{sw}}^{2}$ is the momentum flux of the incident particles, $C_{\mathrm{sw}, i}$ describes the momentum transfer cross section for impinging $i$ th particles, which is analogous to the radiationpressure cross section $C_{\mathrm{pr}}$. In what follows, we derive an analytical expression of $C_{\mathrm{sw}, i}$ accounting for the passage of ions through dust grains.

The stopping power of ions with kinetic energy $E$ in a solid of molecular number density $n_{\mathrm{s}}$ is approximated by

$-\frac{1}{n_{\mathrm{s}}} \frac{\mathrm{d} E}{\mathrm{~d} x}=S^{(1)}\left(\frac{E / m_{\mathrm{sw}}}{1 \mathrm{keV} / \mathrm{amu}}\right)^{\alpha}$,

where $x$ is the traveling distance of the ion in the solid, $S^{(1)}$ the stopping cross section at energy of $1 \mathrm{keV} / \mathrm{amu}$, and $m_{\mathrm{sw}}$ the mass of the ion. The exponent $\alpha$ is assumed to be 0.5 in agreement with most models and experimental results for $E \ll$ $100 \mathrm{keV}$ (Eder et al. 1997; Møller et al. 2002). The projected range $l(E)$ of an ion having initial energy $E_{0}$ is calculated to be:

$l\left(E_{0}\right)=-\int_{0}^{E_{0}} \frac{\mathrm{d} E}{\mathrm{~d} E / \mathrm{d} x}=\frac{2 E_{0}}{\left.(-\mathrm{d} E / \mathrm{d} x)\right|_{E=E_{0}}}$.

Table 1 shows the projected ranges of protons and $\alpha$-particles with initial energy $1 \mathrm{keV} / \mathrm{amu}$ for $\mathrm{SiO}_{2}$ and carbon, which are taken as plausible analogue materials for dust in the solar system. Since Eq. (3) with $\alpha=0.5$ reduces to $-\mathrm{d} p / \mathrm{d} x=$ const., where $p$ is the momentum of the ion, we have

$\frac{p_{0}-p}{p_{0}}=\frac{x}{l\left(p_{0}\right)}$

where $p_{0}=\sqrt{2 m_{\mathrm{sw}} E_{0}}$ is the initial momentum of an incident ion. This relation indicates that the momentum transfer is proportional to the traveling distance $x$ in the grain.

On the basis of Eq. (5), we derive an expression of $C_{\mathrm{sw}, i}$ by summing up the momentum loss rate of solar-wind particles hitting on the grain. The reflection efficiencies of protons and $\alpha$-particles with typical solar-wind velocity are negligibly small (Colligon 1961; Hagstrum 1961). Here we consider a spherical solid grain for simplicity, although actual interplanetary grains may be fluffy. Our study can be extended to estimate the momentum transfer cross section of fluffy dust, but this is left for a future study.

First we consider the case that the projected range $l\left(p_{0}\right)$ of solar-wind particles of momentum $p_{0}$ is larger than the diameter $2 a$ of a grain; all hitting particles pass through the grain. As illustrated in Fig. 1, the momentum loss of an impinging particle during its passage through the grain with impact parameter $a \sin \theta$ is given by

$p_{0}-p=\frac{2 p_{0} a \cos \theta}{l\left(p_{0}\right)}=\frac{2 m_{\mathrm{sw}} v_{\mathrm{sw}} a \cos \theta}{l\left(p_{0}\right)}$.

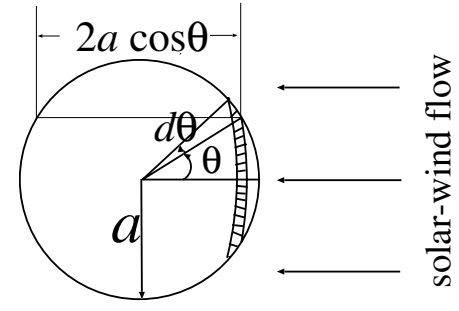

Fig. 1. Geometry of a spherical grain irradiated by solar wind.

The number of solar-wind particles impacting on a ring surface of radius $a \sin \theta$ and width $a \cos \theta \mathrm{d} \theta$ perpendicular to the solarwind-momentum vector per unit time is given by

$\mathrm{d} \dot{N}=2 \pi a^{2} n v_{\text {sw }} \sin \theta \cos \theta \mathrm{d} \theta$

where $n$ is the number density of solar-wind particles. The momentum transfer rate is given by

$\left(p_{0}-p\right) \mathrm{d} \dot{N}=\frac{4 \pi a^{3} \rho v_{\mathrm{sw}}^{2}}{l\left(p_{0}\right)} \cos ^{2} \theta \sin \theta \mathrm{d} \theta$,

where $\rho=n m_{\mathrm{sw}}$. The force exerted on the grain is

$f=\int_{\theta=0}^{\pi / 2}\left(p_{0}-p\right) \mathrm{d} \dot{N}=\frac{4 \pi a^{3} \rho v_{\mathrm{sw}}^{2}}{3 l\left(p_{0}\right)}$.

As a result, we have

$C_{\mathrm{sw}, i}=\pi a^{2}\left[\frac{2}{3}\left(\frac{2 a}{l\left(p_{0}\right)}\right)\right] \quad$ for $\quad 2 a \leq l\left(p_{0}\right)$.

Next we consider a large grain with $2 a>l\left(p_{0}\right)$. Solar-wind particles with impact parameter smaller than $a \sin \theta_{0}$ are absorbed in the grain and exert a force

$f_{1}=\rho v_{\mathrm{sw}}^{2} \pi a^{2} \sin ^{2} \theta_{0}$,

where $\theta_{0}$ is given as

$2 a \cos \theta_{0}=l\left(p_{0}\right)$.

On the other hand, solar-wind particles with impact parameter larger than $a \sin \theta_{0}$ deposit a fraction of their momentum in the grain, and exert a force

$f_{2}=\frac{4 \pi a^{3} \rho v_{\mathrm{sw}}^{2}}{l\left(p_{0}\right)} \int_{\theta_{0}}^{\pi / 2} \cos ^{2} \theta \sin \theta \mathrm{d} \theta=\frac{4 \pi a^{3} \rho v_{\mathrm{sw}}^{2}}{3 l\left(p_{0}\right)} \cos ^{3} \theta_{0}$.

Thus, the total force exerted to a grain is given by

$f=f_{1}+f_{2}=\rho v_{\mathrm{sw}}^{2} \pi a^{2}\left(\sin ^{2} \theta_{0}+\frac{4 a}{3 l\left(p_{0}\right)} \cos ^{3} \theta_{0}\right)$.

Using Eq. (12), we obtain

$C_{\mathrm{sw}, i}=\pi a^{2}\left[1-\frac{1}{3}\left(\frac{l\left(p_{0}\right)}{2 a}\right)^{2}\right] \quad$ for $\quad 2 a>l\left(p_{0}\right)$.

In summary, the momentum transfer cross section is

$C_{\mathrm{sw}, i}=\pi a^{2}\left[\frac{2}{3} X U(1-X)+\left(1-\frac{1}{3 X^{2}}\right) U(X-1)\right]$,

where $X=2 a / l\left(p_{0}\right)$ is the "size parameter" and $U$ is the Heaviside unit step function. Note that the first and second terms are smoothly connected at $X=1$. 


\subsection{Velocity-distribution function}

Solar-wind particles are neutralized and loose momentum along their paths in a grain. As a result, there arises a velocity distribution of solar-wind particles that have passed through a grain, even if we assume them to initially have identical velocities. The velocity distribution is proportional to $\mathrm{d} \dot{N}$ given by Eq. (7) and one arrives at the distribution function by eliminating $\theta$ in $\mathrm{d} \dot{N}$ with the use of Eq. (6) and consideration of Eq. (12) for large grains and by expressing $p$ in terms of the velocity $v=v_{0}-\Delta v$ :

$f(v) \mathrm{d} v=$ const $\cdot\left(v_{0}-v\right) \mathrm{d} v$,

where $v_{0}=p_{0} / m_{\mathrm{sw}}$, and $v_{\min } \leq v \leq v_{0}$ with $v_{\min }=0$ for $2 a>$ $l\left(v_{0}\right)$ and $v_{\min }=v_{0}\left[1-2 a / l\left(v_{0}\right)\right]$ for $2 a<l\left(v_{0}\right)$. Equation (17) indicates a large population at low velocities.

\section{Numerical evaluations}

As to the solar-wind parameters, we use the following averaged values (Neugebauer 2001): $v_{\mathrm{sw}}=4.5 \times 10^{7} \mathrm{~cm} \mathrm{~s}^{-1}$ for the heliocentric velocity and $n(r)=n_{0}(r / 1 \mathrm{AU})^{-2}$ for the number density where $n_{0}=6 \mathrm{~cm}^{-3}$. Above parameters reflect ecliptic conditions that are appropriate for the majority of interplanetary dust grains. At higher heliographic latitudes, the solar-wind speed is faster and the particle density decreases: $v_{\text {sw }}=7.5 \times 10^{7} \mathrm{~cm} \mathrm{~s}^{-1}, n_{0}=3.5 \mathrm{~cm}^{-3}$. We take the number ratio of protons and $\alpha$-particles to be $1: 0.05$ and neglect heavier particles (Neugebauer 2001). Solar-cycle variations of the solar-wind parameters are not considered here, because the time scales of the dynamical evolution of dust grains due to the pseudo PR drag are long compared to the solar cycle.

\subsection{Radial pressure on dust grains}

We calculate the radial solar-wind pressure $F_{\text {sw }}$ on dust grains in ecliptic orbits around the sun as a function of grain radius. Solid lines in Fig. 2 (see the left axes) show the ratios of the solar-wind pressure $F_{\mathrm{sw}}$ to solar-radiation pressure $F_{\mathrm{ph}}$ computed by Mie theory for $\mathrm{SiO}_{2}$ and carbon grains (cf., Mukai \& Mukai 1973; Kimura \& Mann 1999). Figure 2 illustrates the effect of the fractional momentum transfer of the solarwind particles to the grain in comparison to the perfect momentum transfer of the particles assumed in previous studies corresponding to $C_{\mathrm{sw}, i}=\pi a^{2}$ (dotted lines). For grains larger than $0.1 \mu \mathrm{m}$, the solar-wind pressure is $\sim 10^{-3}$ of the radiation pressure and the ratio of solar-wind pressure to radiation pressure is nearly constant because both forces are approximately proportional to the geometrical cross section of the grains. For grains smaller than $0.1 \mu \mathrm{m}$, the ratio depends on the size and material of the grains but remains smaller than unity. At high heliographic latitudes, the solar-wind pressure is stronger for large grains of $X \gg 1$ due to larger momentum flux of the solar wind, but approximately constant with latitudes for small grains of $X<1$ because the effect of the larger momentum flux is canceled out by the small-particle effect.
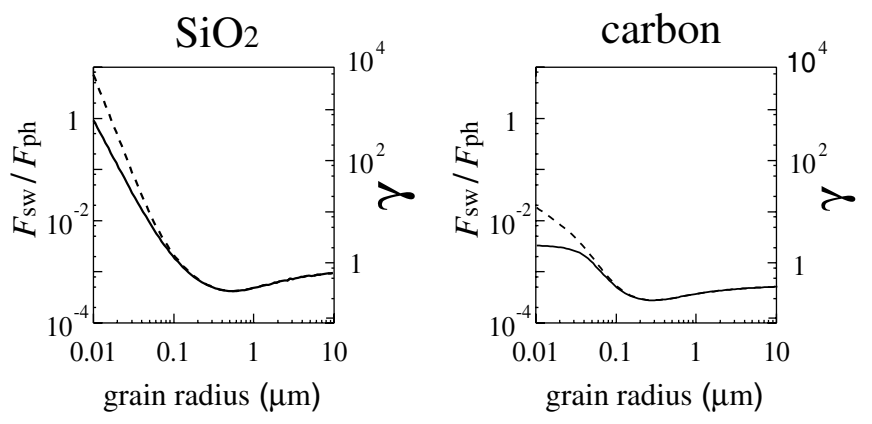

Fig. 2. Ratio $F_{\mathrm{sw}} / F_{\mathrm{ph}}$ of solar-wind pressure to electromagnetic radiation pressure (left scales) and ratio $\gamma$ of the pseudo PR drag to the photon PR drag (right scales) as a function of grain radius taking into account the fractional momentum loss (solid line) for $\mathrm{SiO}_{2}$ (left panel) and for carbon (right panel). For comparison, the dotted lines indicate the cases that all impinging particles are completely absorbed.

\subsection{Tangential drag force on dust grains}

The second term on the right-hand side of Eq. (1) expresses the pseudo PR drag which causes dust grains to spiral into the sun. The ratio $\gamma$ of the pseudo PR drag to the PR drag is given by $\gamma=\left(F_{\mathrm{sw}} / F_{\mathrm{ph}}\right)\left(c / v_{\mathrm{sw}}\right)$ where the factor $c / v_{\mathrm{sw}}$ comes from the difference of the aberration angles for solar wind and light. Figure 2 also gives the $\gamma$ ratios for $\mathrm{SiO}_{2}$ and carbon grains (see the right axes). The pseudo PR drag exceeds the PR drag up to two orders of magnitude for grains smaller than $0.1 \mu \mathrm{m}$ although the passage of solar-wind particles limits the relative importance of the pseudo PR drag. Because the pseudo PR drag does not strongly depend on the materials of grains, the difference in the values between $\mathrm{SiO}_{2}$ and carbon comes mainly from the material dependence of the PR drag. The $\gamma$ ratios shown in Fig. 2 holds at any heliographic latitude for large grains of $X \gg 1$ owing to the fact that the mass flux of solar wind is nearly constant with latitude (Phillips et al. 1995). For small grains $X \ll 1$, the fast solar wind at high latitudes weakens the pseudo PR drag. The varying pseudo PR drag with latitudes influences the dynamical evolution of interplanetary dust (Banaszkiewicz et al. 1994; Fahr et al. 1995).

\subsection{Velocity distribution}

Figure 3 shows the velocity distribution function $f(v)$ of protons after passage through $\mathrm{SiO}_{2}$ grains with radii of $a=100$, 250 , and $>500 \AA$ for $v_{0}=4.5 \times 10^{7} \mathrm{~cm} \mathrm{~s}^{-1}$. The distribution function increases linearly with decreasing velocity and has a low-velocity cutoff $v_{\min }$. Note that $v_{\min }$ decreases with grain radius $a$, but $v_{\text {min }}=0$ for $a>500 \AA$, implying that large grains produce a wide velocity distribution.

\section{Discussion}

The dependence of momentum transfer cross section $C_{\mathrm{sw}}$ here derived on the grain radius is analogous to that of the radiationpressure cross section $C_{\mathrm{pr}}$. For a small grain $(X<1), C_{\mathrm{sw}}$ is proportional to the volume of the grain because the number of incident particles depends on the grain cross section and the momentum loss depends on the mean thickness of the grain. 


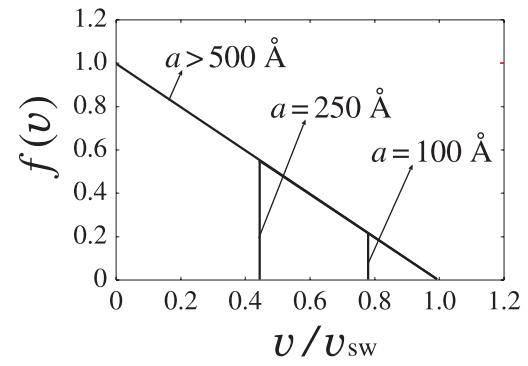

Fig. 3. Velocity distribution $f(v)$ of protons after passage through $\mathrm{SiO}_{2}$ grains with radii of $a=100,250$, and $>500 \AA$. Initial velocity of protons is taken to be $v_{\mathrm{sw}}=4.5 \times 10^{7} \mathrm{~cm} \mathrm{~s}^{-1}$.

For larger grains $(X \geq 1), C_{\mathrm{sw}}$ approaches the geometric cross section because almost all of the incident particles are absorbed by the grains. The former and the latter correspond to $C_{\mathrm{pr}}$ in the Rayleigh scattering and the geometrical optics, respectively.

The present derivation of the expression for the momentum transfer cross section enables us to more correctly estimate the solar-wind pressure. The magnitude of solar-wind pressure is found to always stay below that of solar-radiation pressure for grains of sizes $\geq 0.01 \mu \mathrm{m}$, as opposed to the previous work by Mukai \& Yamamoto (1982) who suggested the solar-wind pressure to exceed radiation pressure by one order of magnitude for very small grains of $\sim 0.01 \mu \mathrm{m}$ (Fig. 2). We conclude that the direct solar-wind pressure is negligible for most sizes $(>0.01 \mu \mathrm{m})$ and compositions of grains.

Unlike the direct solar-wind pressure, the pseudo PR effect dominates the PR effect for grains of sizes $<0.1 \mu \mathrm{m}$ and is important even for larger grains (Fig. 2). Previous estimates of the pseudo PR effect are qualitatively consistent with our results, but quantitatively different for small grains. We note that neglecting the passage of solar-wind particles through dust grains greatly underestimates the lifetime of very small grains.

We have neglected some effects considered in Mukai \& Yamamoto (1982) when calculating the solar-wind forces. The effects of sputtering of surface molecules increase the forces by a factor of $<1.4$ at $1 \mathrm{AU}$ compared to the values given in this paper. With a typical solar-wind speed $\left(\sim 400-800 \mathrm{~km} \mathrm{~s}^{-1}\right)$ and temperature $\left(\sim 10^{4} \mathrm{~K}\right)$, thermal velocity dispersion of particles increases the forces by $<1.03$. Our results slightly underestimate the forces acting on grains in prograde, while they overestimate the forces for retrograde orbits. Even taking into account the above mentioned effects, our estimates for the solar-wind forces lead qualitatively to the same results.

The velocity distribution shown in Fig. 3 has implications for the current discussion of the production of pick-up ions in the inner solar system. Wimmer-Schweingruber \& Bochsler (2003) suggested that the passage of solar-wind particles through tiny dust grains generates pick-up ions near the sun.
However, as pointed out by Mann et al. (2004), neither does the required density of small grains agree with dust-collisionalevolution models of Ishimoto \& Mann (1999) nor does this work discuss the velocity distribution of the species that pass through the grains. Our results indicate that numbers of lowvelocity protons arises from large grains with radii $a>500 \AA$, while small grains of $a<100 \AA$ decelerate impinging protons at most to $80 \%$ solar-wind speed. Heavier ions require larger grains to be sufficiently decelerated. Therefore, estimating the contribution of tiny grains to the production of pick-up ions requires detailed consideration of the velocity distribution of solar-wind particles passing through the grains.

Acknowledgements. T.M. thanks $D A A D$ for financial support to stay at IfP WWU Münster. Research at IfP is supported by DLR under the projects "Kosmischer Staub" (RD-RX-50OO0101-ZA) and "Mikro-Impakte" (RD-RX-50OO0203). T.Y. acknowledges the support by the grant from the Inst. of Low Temp. Sci., Hokkaido Univ. and the grants-in-aid from the JSPS.

\section{References}

Banaszkiewicz, M., Fahr, H. J., \& Scherer, K. 1994, Icarus, 107, 358 Banks, P. M. 1971, J. Geophys. Res., 76, 4341

Bauer, P., Golser, R., Semrad, D., et al. 1998, Nucl. Instrum. Methods Phys. Res. B, 136-138, 103

Burns, J. A., Lamy, P. L., \& Soter, S. 1979, Icarus, 40, 1

Colligon, J. S. 1961, Vacuum, 11, 272

Eder, K., Semrad, D., Bauer, P., et al. 1997, Phys. Rev. Lett., 79, 4112

Fahr, H. J., Ripken, H. W., \& Lamy, G. 1981, A\&A, 102, 359

Fahr, H. J., Scherer, K., \& Banaszkiewicz, M. 1995, Planet. Space Sci., 43,301

Gloeckler, G., \& Geiss, J. 2001, Space Sci. Rev., 97, 169

Gustafson, B. A. S. 1994, Annu. Rev. Earth Planet. Sci., 22, 553

Hagstrum, H. D. 1961, Phys. Rev., 123, 758

Ishimoto, H., \& Mann, I. 1999, Planet. Space Sci., 47, 225

Kimura, H., \& Mann, I. 1999, in Meteoroids 1998, ed. W. J. Baggaley, \& V. Porubcan (Bratislava: Astron. Inst., Slovak Acad. Sci.), 283

Mann, I., Kimura, H., Biesecker, D. A., et al. 2004, Space Sci. Rev., 110, 269

Mikheev, S., Ryzhov, Y., Shkarban, I., \& Yurasova, V. 1993, Nucl. Instrum. Methods Phys. Res. B, 78, 86

Møller, S. P., Csete, A., Ichioka, T., et al. 2002, Phys. Rev. Lett., 88 , 193201

Mukai, T., \& Mukai, S. 1973, PASJ, 25, 481

Mukai, T., \& Yamamoto, T. 1982, A\&A, 107, 97

Neugebauer, M. 2001, in The Heliosphere Near Solar Minimum, ed. A. Balogh, R. G. Marsden, \& E. J. Smith (Chichester: Springer), 43 Phillips, J. L., Bame, S. J., Barnes, A., et al. 1995, Geophys. Res. Lett., 22, 3301

Whipple, F. L. 1955, ApJ, 121, 750

Wimmer-Schweingruber, R., \& Bochsler, P. 2003, Geophys. Res. Lett., 30, 49 